\title{
Unusual suspects: Real-time physiological evaluation of stressors during laparoscopic donor nephrectomy
}

Claire Wilson $^{1}$; Saad Chahine ${ }^{2}$; Sayra Cristancho ${ }^{1}$; Shahid Aquil ${ }^{1}$; Moaath Mandurah ${ }^{3}$; Max Levine $^{3}$; Alp Sener ${ }^{3}$

${ }^{1}$ Department of Surgery, Schulich School of Medicine and Dentistry, Western University, London, ON, Canada; ${ }^{2}$ Queen's University, Kingston, ON, Canada; ${ }^{3}$ London Health Sciences Centre, London, ON, Canada

Funding: This work was supported by a Faculty Support for Research in Education Grant from the Schulich School of Medicine and Dentistry (\#41624).

Cite as: Wilson $\mathrm{C}$, Chahine $\mathrm{S}$, Cristancho $\mathrm{S}$, et al. Unusual suspects: Real-time physiological evaluation of stressors during laparoscopic donor nephrectomy. Can Urol Assoc J 2020

September 28; Epub ahead of print. http://dx.doi.org/10.5489/cuaj.6647

Published online September 28, 2020

$* * *$

\section{Abstract}

Introduction: The purpose of this study was to document the variability of faculty surgeon electrodermal activity (EDA) peaks during laparoscopic donor nephrectomy (LDN) to determine the effect of case difficulty and learner expertise on the stress response.

Methods: EDA for a single faculty surgeon was captured over 15 LDN cases using an Empatica E4 wristband. During each case, one of three transplant fellows (novice, intermediate, or expert level LDN expertise) participated. Difficulty was rated preoperatively as "low/moderate/high" by the faculty. EDA peaks were collected and analyzed; the frequency and magnitude of EDA peaks, case difficulty, and fellow expertise were compared using a two-way factorial ANOVA. Results: The main effects of learner expertise $(\mathrm{F}[2,308]=11.27, \mathrm{p}<0.001)$ and difficulty rating $(\mathrm{F}[2,414]=15.13, \mathrm{p}<0.001)$ were significant. The interaction between difficulty and expertise on faculty EDA peaks was also significant $(\mathrm{F}[3,391]=14.29, \mathrm{p}<0.001)$. The novice fellow resulted in higher faculty EDA levels compared to intermediate and expert fellows on low-difficulty cases, but not moderate- or high-difficulty cases.

Conclusions: This is the first report examining faculty surgeon EDA across cases of varying difficulty and varying learner expertise during a high-stakes operation. EDA levels were inversely proportional to the expertise of the learner and case difficulty, suggestive of a significant impact of learner autonomy on faculty stress response. 


\section{Introduction}

Stress during surgery is an important physiological response; a moderate amount of stress can be productive, while too much can be detrimental. ${ }^{1,2}$ Excessive intraoperative stress can result in lower performance in potentially lifesaving procedures, ${ }^{3}$ and can also impair communication, ${ }^{4,5}$ which is particularly critical for the faculty-trainee relationship. While a number of studies have examined the sources and outcomes of stress in the OR, very few studies have examined moderating contextual factors that may impact stress variability.

Faculty in the OR are actively maintaining a balance between the best methods to teach, whilst considering the needs of the learner and optimal outcomes for patients. Previous research with cardiac surgeons has indicated that the faculty surgeon is more stressed when observing or assisting a resident than when the surgeon is performing the operation himself. ${ }^{6,7}$ While these studies have demonstrated that trainee participation is often a source of faculty stress, these findings have yet to be explored across varying levels of trainee expertise.

Another contextual factor that has rarely been explored in relation to surgeon stress is perceived case difficulty. Recently, Quick and colleagues ${ }^{8}$ studied the effects of stress, case difficulty, and learner expertise on operative time during laparoscopic cholecystectomies. They found operative time increased with case difficulty, and this finding was consistent across all levels of trainee expertise; however, this study did not examine how these factors impacted faculty surgeon stress.

Studies examining outcomes of stress in the OR have primarily used heart rate, heart rate variability, and electrodermal response as objective measures of stress. ${ }^{5,9}$ Electrodermal activity (EDA) metrics capture the change in sympathetic arousal that is influenced by cognitive and emotional states and it is not confounded by activity within the parasympathetic system. ${ }^{10}$ Recently, EDA activity along with psychological inventories were used to measure nurse stress and burnout longitudinally. High moments of stress can be described as EDA peaks which are estimated through a mathematical algorithm that considers heightened levels of skin conductivity, temperature, movement and blood volume pulse. ${ }^{11,12}$ The algorithms have also been validated in other fields of research such as computer science and machine learning. ${ }^{13,14}$

Thus, the aim of this study is to explore the impact of two infrequently studied factorscase difficulty and trainee expertise - on faculty surgeon stress during living donor laparoscopic nephrectomies. EDA peak magnitude and frequency are analyzed as a measure of objective faculty intraoperative stress. The interaction between case difficulty and learner expertise on faculty stress will be explored across 15 laparoscopic living donor nephrectomies. It is hypothesized that operating with more novice learners, particularly during subjectively difficult cases, will result in increased faculty surgeon stress as indicated by heightened frequency and magnitude of EDA peaks. 


\section{Methods}

\section{Participants and procedure}

This study received approval from Western University's Health Sciences Research Ethics Board. This study utilized a single case, multiple baseline design. The multiple baseline method is a preferred approach of capturing changes in behaviour in one or more persons. The method is flexible and allows for settings and contexts to vary. ${ }^{15}$ Due to their flexibility and practicality, multiple baseline designs are frequently used in educational and clinical settings when studying an individual or small number of cases. ${ }^{16}$ The present study examines multiple baselines of faculty stress (behaviour) across varying contexts (operating alongside different learners with varying levels of expertise). EDA data for a single faculty surgeon with 10 years of experience were captured over 15 laparoscopic living donor nephrectomy transplant surgeries at a teaching hospital in Ontario, Canada. EDA data were collected through the use of an Empatica E4 ${ }^{17}$ wristband. The wristbands were worn according to the manufacturer's instructions on the dominant wrist underneath the surgical gown. The wristband was turned on and recording began just prior to the surgeons scrubbing in for surgery. The surgeons scrubbed with an alcohol-based surgical scrub which the wristband is impervious to, and the sterile gloves and gown were worn over top of the device. Data were collected for the entire length of the surgery, and recording was stopped when the surgeon disrobed at the end. Data were uploaded automatically to the online Empatica data manager (www.empatica.com).

\section{Learner expertise}

Three transplant fellows of varying expertise - novice, intermediate, expert - participated across the 15 cases. The level of expertise for each fellow was determined by the faculty surgeon based on previous laparoscopic experience, and their performance on similar cases. Across the 15 cases, the novice fellow participated in 4 cases, the intermediate fellow participated in 5 cases, and the expert fellow participated in 6 cases.

\section{Case difficulty}

Prior to each surgery, the faculty surgeon rated the case as "low", "moderate", or "high" difficulty. Ratings were based primarily on pre-operative scans and patient characteristics. The criteria for the difficulty ratings were as follows: low difficulty cases consisted of standard anatomy (i.e., single artery/vein), and left side donation; moderately difficult cases consisted of previous inflammation in the relevant area causing fibrosis, and right side donation; high difficulty cases included those with multiple renal arteries and veins, having previously received surgery in the area, and right side donation. Case difficulty ratings were updated post-surgery to reflect the true case difficulty after taking into account interoperative case complexities. 


\section{Stress}

Faculty surgeon stress was assessed by the frequency and magnitude of EDA peaks measured using the Empatica E4 wristband. The Empatica E4 collects a variety of physiological indicators including EDA, blood volume pulse, and peripheral skin temperature, ${ }^{17}$ however only EDA data were analyzed for the present study as a measure of stress. At the end of each surgery, the wristband data were automatically uploaded to the Empatica online data manager. EDA peaks were identified using the EDA Explorer software with default threshold values (http://edaexplorer.media.mit.edu/). ${ }^{12}$

\section{Data analysis}

Descriptive statistics for the frequency and magnitude of EDA peaks (measured in micro Siemens $\mu \mathrm{S}$ ), were conducted for case difficulty and transplant fellow expertise. A two-way factorial ANOVA was conducted to compare the main effects of case difficulty, and transplant fellow expertise, as well as the interaction on the magnitude of EDA peaks.

\section{Results}

The mean faculty EDA peaks for cases of varying difficulty level and cases with learners of varying expertise can be found in Tables 1 and 2 respectively. The main effect of learner expertise $F(2,308)=11.27, p<.001 ; \eta^{2}=.010$, and difficulty rating $F(2,414)=15.13, p<.001$; $\eta^{2}=.013$ were significant. The interaction of case difficulty and learner expertise on surgeon EDA peaks was also significant $F(3,391)=14.29, p<.001 ; \eta^{2}=.018$.

Tukey post hoc analysis reveals that overall, the intermediate learner resulted in significantly higher mean EDA $(10.18 \pm 6.00 \mu \mathrm{S}, p<.001)$ compared to the expert $(8.80 \pm$ $5.02 \mu \mathrm{S}, p<.001)$ and novice fellows $(3.34 \pm .82 \mu \mathrm{S}, p<.001)$. Further, high difficulty cases $(11.43 \pm 5.66 \mu \mathrm{S}, p,<.001)$ resulted in significantly higher mean EDA compared to moderate $(9.32 \pm 5.57 \mu \mathrm{S}, p<.001)$ and low $(2.97 \pm 1.12 \mu \mathrm{S}, p<.001)$ difficulty cases. Figure 1 shows elevated faculty EDA in moderate/high difficulty cases when the intermediate and expert fellows were involved. The novice fellow did not result in higher levels of EDA on moderate/difficult cases but did result in higher faculty EDA levels compared to the intermediate and expert fellows on low difficulty cases.

\section{Discussion}

The goal of this study was to examine the interaction between faculty surgeon stress, case difficulty, and learner expertise, and the results present both confirming and unexpected findings. As may be expected, case difficulty independently contributed to surgeon stress such that more difficult cases resulted in greater stress. Learner expertise also independently contributed to surgeon stress, but in an unexpected way with the intermediate learner resulting in greater surgeon stress compared to both the novice and expert learner. We speculate that this may be due to the uncertain nature of the intermediate learner's level of ability and faculty uncertainty in 
how much leeway to permit an intermediate learner. The significant interaction of case difficulty and learner ability was also unexpected - more difficult cases completed with the more experienced learner resulted in the greatest stress. These findings are in contrast to our hypothesis that the more novice learner would increase surgeon stress during difficult cases.

One explanation for these unexpected findings may be the amount of control the faculty surgeon maintains when operating with learners of varying expertise. Kuhn and colleagues ${ }^{6}$ found that the process of relinquishing control while still maintaining responsibility, results in faculty experiencing heightened stress in the OR. Therefore, to alleviate this stress, perhaps during high difficulty surgeries with a novice learner, the faculty surgeon may spend more time conducting the procedure due to lack of learner experience. Comparatively, the faculty may permit novice learners to participate more during low difficulty cases where the risk appears lower. This might explain why novice learners resulted in the greatest stress during low difficulty surgeries, but minimal stress during high difficulty cases. Similarly, due to their increased experience, more expert learners may be given greater opportunity to participate during moderate and high difficulty cases, resulting in less faculty participation. Perhaps it is the surgeon's level of control (or lack thereof) that is influencing stress levels. These findings complement previous research that have found faculty surgeons stress levels increased when observing residents, compared to when they were operating themselves. ${ }^{6,7}$

However, the implicit assumption that more experienced trainees may be better equipped to deal with more difficult cases, may not be entirely accurate. Recent research examining trainee performance during laparoscopic cholecystectomies found that for all levels of trainees, difficult cases were more complex across all phases of the operation, including the initial "simpler" stages. Furthermore, there were minimal differences in operating time between novice and more senior trainees. ${ }^{8}$ Therefore, discrepancies between faculty expectation and trainee performance when experienced trainees are operating, may also have an impact on faculty surgeon stress. However, further research is required to determine the differential effects of expectations and trainee performance on faculty stress.

This study suggests that learner level of expertise significantly contributes to faculty surgeon stress in the OR, particularly during more difficult cases. This knowledge may be useful for faculty when considering the best way to maintain a balance between teaching and ensuring optimal outcomes for patients. Stress management and resilience training is demonstrably useful in decreasing surgeon stress and anxiety generally, ${ }^{18,19}$ and therefore may serve as a useful tool to help faculty manage stress, and better support trainees in surgical settings, although this needs to be explored further.

This study is limited to the findings of a single faculty surgeon and a limited number of trainees. Future research may consider including a greater number of faculty to investigate how recent research on individual surgical preferences during laparoscopic nephrectomy procedures ${ }^{20}$ are associated with stress responses. Furthermore, previous research has revealed it is 
challenging to predict actual case difficulty, particularly in laparoscopic nephrectomies. ${ }^{21}$ However surgeon perceived difficulty may nevertheless affect stress levels, and discrepancies between actual and perceived case difficulty should be explored further. With a larger sample and more institutions, we may better understand the generalization of stress responses that are based on the interaction of case difficulty and trainee expertise. Additionally while EDA is increasingly being recognized as a valuable indicator of autonomic sympathetic arousal, EDA data may be affected by a number of external variables including room temperature, humidity, and medications. ${ }^{22}$ Although technology is advancing and EDA is often used as an indicator of general stress, we are unable to determine if peaks are due specifically to emotional arousal, cognitive arousal, or attention. Lastly, other external factors such as the experience of the nursing team and technical difficulties with equipment could also influence surgeon stress and should be considered as a possible limitation of this study. In future research, the addition of observational data may help elucidate the interaction between case difficulty, trainee expertise, and surgeon stress.

\section{Conclusions}

In summary, this study presents a novel finding of the relationship between case difficulty and trainee expertise on peaks of stress. What we can confirm from our data is that case difficulty and learner expertise interact such that faculty surgeon stress is greatest with an expert learner during high difficulty cases. Yet more research is needed to better understand the generalization of these findings and how different surgeons' stress responses vary with different learners in different contexts. While some stress is beneficial to performance and learning, too much stress may impede the learning process as well as potentially comprise patient safety.

Therefore, it is important to understand the contextual factors that contribute to increased stress during surgical procedures. 


\section{References}

1. Ng R, Chahine S, Lanting B. Unpacking the literature on stress and resiliency: A narrative review focused on learners in the operating room. J Surg Educ 2019;76:343-53.

2. LeBlanc VR. The effects of acute stress on performance: Implications for health professions education. Acad Med 2009;84:25-33.

3. Harvey A, Bandiera G, Nathens AB. Impact of stress on resident performance in simulated trauma scenarios. J Trauma Acute Care Surg 2012;72:497-503.

4. Wetzel CM, Kneebone RL, Woloshynowych M. et al. The effects of stress on surgical performance. Am J Surg 2006(1);191:5-10.

5. Arora S, Sevdalis N, Nestel D. The impact of stress on surgical performance: A systematic review of the literature. Surgery 2010;147:318-30.

6. Kuhn EW, Choi YH, Schönherr M, et al. Intraoperative stress in cardiac surgery: Attendings versus residents. J Surg Res 2013;182:43-49.

7. Song MH, Tokuda Y, Nakayama T. Intraoperative heart rate variability of a cardiac surgeon himself in coronary artery bypass grafting surgery. Interact Cardiovasc Thorac Surg 2009;8:639-41.

8. Quick JA, Bukoski AD, Doty J, et al. Case difficulty, postgraduate year, and resident surgeon stress: Effects on operative times. J Surg Educ 2019;76:354-61.

9. Phitayakorn R, Minehart RD, Pian-Smith MC. Practicality of using galvanic skin response to measure intraoperative physiologic autonomic activation in operating room team members. Surgery 2015;158:1415-20.

10. Critchley HD. Electrodermal responses: What happens in the brain. Neuroscientist 2002;8:132-42.

11. De Looff P, Didden R, Embregts P. Burnout symptoms in forensic mental health nurses: Results from a longitudinal study. Int J Meant Health Nurs 2019;28:306-17.

12. Taylor S, Jaques N, Chen W. Automatic identification of artifacts in electrodermal activity data. Paper presented at Annual International Conference of the IEEE Engineering in Medicine and Biology Society; August, 2015. Milan, Italy.

13. Jaques N, Taylor S, Azaria A. Predicting students' happiness from physiology, phone, mobility, and behavioral data. Paper presented at International Conference on Affective Computing and Intelligent Interaction; September, 2015. Xian, China.

14. Jaques N, Taylor S, Sano A. Predicting tomorrow's mood, health, and stress level using personalized multitask learning and domain adaptation. Paper presented at Workshop on Artificial Intelligence in Affective Computing; August 2017. Melbourne, Australia.

15. Murphy RJ, Bryan AJ. Multiple-baseline and multiple-probe designs: Practical alternatives for special education assessment and evaluation. J Spec Educ 1980;14:32535.

16. Gast DL, Lloyd BP, Ledford JR. Multiple baseline and multiple probe designs. In: Ledford JR, Gast DL, eds. Single Case Research Methodology. $3^{\text {rd }}$ ed. New York: Routledge. 2018. p 239-281.

17. E4 Wristband User's Manual 20150608. Empatica Milan Italy, pp. 5-16, 2015. 
18. Sood A, Sharma V, Schroeder DR. Stress Management and Resiliency Training (SMART) program among department of radiology faculty: A pilot randomized clinical trial. Explore (NY) 2014;10:358-63.

19. Sood A, Prasad K, Schroeder D. Stress management and resilience training among department of medicine faculty: a pilot randomized clinical trial. J Gen Intern Med 2011;26:858-61.

20. Apramian T, Cristancho S, Sener A. How do thresholds of principle and performance influence surgeon assessments of learner performance? Ann Surg 2018;268:385-90.

21. Ratner LE, Smith P, Montgomery RA. Laparoscopic live donor nephrectomy: Pre-operative assessment of technical difficulty. Clin Transplant 2000;14:427-32.

22. Boucsein W, Fowles DC, Grimnes S. Publication recommendations for electrodermal measurements. Psychophysiology 2012;49:1017-34. 


\section{Figures and Tables}

Fig. 1. Case difficulty and mean EDA peaks for laparoscopic donor nephrectomy cases with transplant fellows of varying expertise. CI: confidence interval; EDA: electrodermal activity.

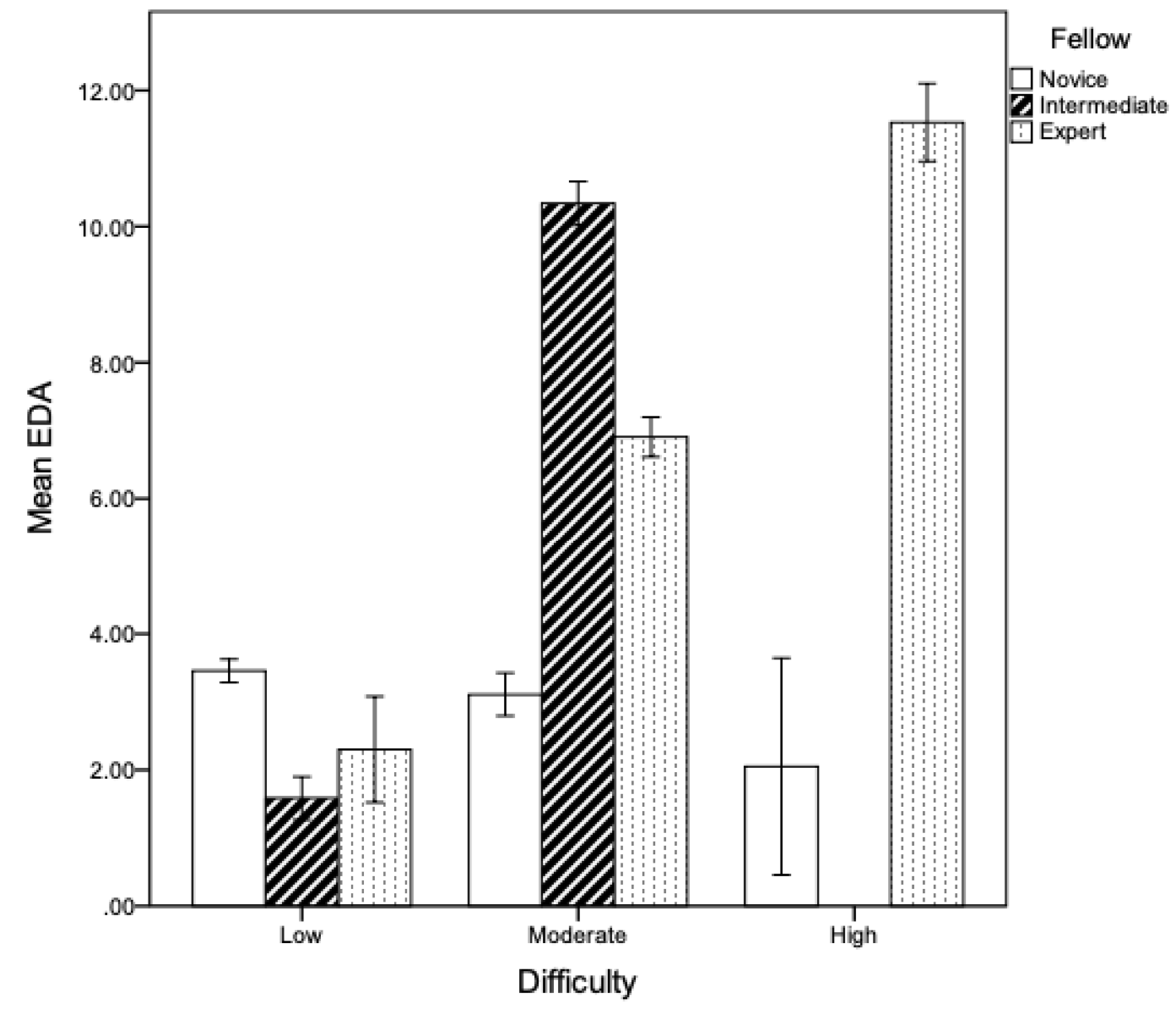

Error bars: $95 \% \mathrm{Cl}$

\begin{tabular}{|c|c|c|c|c|}
\hline Difficulty & $\begin{array}{c}\text { Number of } \\
\text { peaks }\end{array}$ & $\begin{array}{l}\text { EDA peak } \\
\text { mean }(\mu S)\end{array}$ & $\begin{array}{c}\text { EDA peak SD } \\
(\mu S)\end{array}$ & $95 \%$ CI \\
\hline Low & 118 & 2.97 & 1.12 & $2.77,3.18$ \\
\hline Moderate & 1837 & 9.32 & 5.57 & $9.06,9.57$ \\
\hline High & 376 & 11.43 & 5.66 & $10.85,12.00$ \\
\hline
\end{tabular}

CI: confidence interval; EDA: electrodermal activity; SD: standard deviation. 


\begin{tabular}{|l|c|c|c|c|}
\hline \multicolumn{5}{|l|}{ Table 2. Summary statistics for EDA categorized by learner expertise } \\
\hline Fellow & $\begin{array}{c}\text { Number of } \\
\text { peaks }\end{array}$ & $\begin{array}{c}\text { EDA peak } \\
\text { mean }(\boldsymbol{\mu S})\end{array}$ & $\begin{array}{c}\text { EDA peak SD } \\
(\boldsymbol{\mu S})\end{array}$ & $\mathbf{9 5 \%}$ CI \\
\hline Novice & 108 & 3.34 & 0.82 & $3.19,3.50$ \\
\hline Intermediate & 1336 & 10.18 & 6.00 & $9.86,10.50$ \\
\hline Expert & 887 & 8.80 & 5.02 & $8.47,9.13$ \\
\hline
\end{tabular}

CI: confidence interval; EDA: electrodermal activity; SD: standard deviation. 